\title{
Performance Evaluation of Noise Reduction Filters for Color Images through Normalized Color Difference (NCD) Decomposition
}

\author{
Fabrizio Russo \\ Department of Engineering and Architecture, University of Trieste, Via A. Valerio 10, 34127 Trieste, Italy \\ Correspondence should be addressed to Fabrizio Russo; rusfab@univ.trieste.it
}

Received 22 October 2013; Accepted 18 December 2013; Published 22 January 2014

Academic Editors: F. J. Cuevas-de-la-Rosa, A. Nikolaidis, S.-H. Ong, and N. A. Schmid

Copyright (C) 2014 Fabrizio Russo. This is an open access article distributed under the Creative Commons Attribution License, which permits unrestricted use, distribution, and reproduction in any medium, provided the original work is properly cited.

\begin{abstract}
Removing noise without producing image distortion is the challenging goal for any image denoising filter. Thus, the different amounts of residual noise and unwanted blur should be evaluated to analyze the actual performance of a denoising process. In this paper a novel full-reference method for measuring such features in color images is presented. The proposed approach is based on the decomposition of the normalized color difference (NCD) into three components that separately take into account different classes of filtering errors such as the inaccuracy in filtering noise pulses, the inaccuracy in reducing Gaussian noise, and the amount of collateral distortion. Computer simulations show that the proposed method offers significant advantages over other measures of filtering performance in the literature, including the recently proposed vector techniques.
\end{abstract}

\section{Introduction}

It is known that removal of noise and preservation of color/ structural information are very difficult and challenging issues in the design of image denoising filters [1]. Indeed, the quality of a filtered image is typically impaired by the superposition of two different effects: insufficient noise cancellation and unwanted collateral distortion produced by the filtering. Since the different amounts of these effects should separately be taken into account to analyze the behavior of any image denoising technique, the development of appropriate metrics is of paramount importance.

Until recently, the most common methods to evaluate the quality of denoised images were combinations of visual inspection and objective measurements based on the computation of pixelwise differences between the original and the processed image. Typically, the mean squared error (MSE) or the peak signal-to-noise ratio (PSNR) was adopted to measure the noise cancellation, whereas the mean absolute error (MAE) represented the most commonly used metrics to evaluate the edge preservation. All the aforementioned measures are typically evaluated in the RGB coordinate system, that is, the most popular color space for a variety of applications.
In order to deal with the human perception of colors (not adequately described by the RGB space), another kind of metrics, namely, the normalized color difference (NCD), was proposed [1-3]. Such measure is evaluated in the perceptually uniform CIE Luv (or CIE Lab) color spaces in order to appraise the perceptual closeness of a filtered picture to the uncorrupted original. The results of most filters in the literature have been evaluated by resorting to the aforementioned measures or combinations of them [4-19]. Such evaluation techniques, however, have a major drawback. They have limited accuracy in estimating the different filtering features. As already observed for grayscale [20] and color images [21], MSE and MAE cannot accurately measure noise removal and detail preservation, because they cannot separate these features. Although the MAE is more sensitive to distortion than the MSE, it also depends upon the residual noise. On the other hand, even if the MSE is more sensitive to the remaining noise than the MAE, it is affected by the amount of distortion produced by a filter too. Similarly, the NCD takes into account all the filtering errors in perceptually uniform color spaces without distinguishing error contributions caused by filtering distortion or by insufficient noise cancellation. The same limitation also affects metrics that try to estimate the perceived 
quality of an image in the form of a single score [22, 23]. Again, they cannot distinguish between noise cancellation and detail preservation yielded by a filter because different combi nations of image blur and unfiltered noise can lead to the same score. Recently proposed measures such as the vector root mean squared error (VRMSE) are a more appropriate choice because they give a separate evaluation of the mentioned features. A limitation of such techniques, however, is the fact that they work in the RGB [24] and YUV [25] nonuniform color spaces and measure the noise removal and the detail blur in the luminance component of the image only. Furthermore, they cannot address the case of mixed (impulse and Gaussian) noise in color data.

In this paper a new method for measuring the objective quality of filtered images is presented. The proposed approach consists in the decomposition of the NCD into three different components that, respectively, measure how much a filter is good at removing noise pulses, reducing Gaussian noise, and preserving the image details. The method is more accurate than our previous vector techniques and can address the case of mixed noise.

The rest of the paper is organized as follows. Section 2 describes the proposed approach, Section 3 explains how the method works, Section 4 discusses the results of many computer simulations, and, finally, Section 5 reports the conclusion.

\section{The Proposed Method}

The proposed method is specifically designed to address the case of mixed noise. Thus, let us deal with images corrupted by impulse and Gaussian noise as well. Formally, let $\overline{\mathbf{r}}(\mathbf{c})=$ $\left[\bar{r}_{L}(\mathbf{c}), \bar{r}_{u}(\mathbf{c}), \bar{r}_{v}(\mathbf{c})\right]^{T}$ be the vector (in the Luv color space) representing the pixel at spatial position $\mathbf{c}=\left[c_{1}, c_{2}\right]$ in the original noise-free image and let $\overline{\mathbf{y}}(\mathbf{c})=\left[\bar{y}_{L}(\mathbf{c}), \bar{y}_{u}(\mathbf{c}), \vec{y}_{v}(\mathbf{c})\right]^{T}$ be the corresponding pixel in the filtered picture. It is known that the NCD is defined by the following relationship [1]:

$$
\begin{aligned}
\mathrm{NCD}= & \sum_{\mathbf{c} \in D}\left(\left(\bar{y}_{L}(\mathbf{c})-\bar{r}_{L}(\mathbf{c})\right)^{2}+\left(\bar{y}_{u}(\mathbf{c})-\bar{r}_{u}(\mathbf{c})\right)^{2}\right. \\
& \left.+\left(\bar{y}_{v}(\mathbf{c})-\bar{r}_{v}(\mathbf{c})\right)^{2}\right)^{1 / 2} \\
& \times\left(\sum_{\mathbf{c} \in C} \sqrt{\left(\bar{r}_{L}(\mathbf{c})\right)^{2}+\left(\bar{r}_{u}(\mathbf{c})\right)^{2}+\left(\bar{r}_{v}(\mathbf{c})\right)^{2}}\right)^{-1},
\end{aligned}
$$

where $C$ denotes the overall set of coordinates of the processed pixels and $D \equiv C$. In our approach, we want to decompose the NCD into three components $\mathrm{NCD}_{\text {imp }}, \mathrm{NCD}_{\text {gau }}$, and $\mathrm{NCD}_{\text {dis }}$, as follows:

$$
\mathrm{NCD}=\mathrm{NCD}_{\mathrm{imp}}+\mathrm{NCD}_{\text {gau }}+\mathrm{NCD}_{\text {dis }}
$$

where $\mathrm{NCD}_{\text {imp }}$ is the component that deals with partially removed noise pulses, $\mathrm{NCD}_{\text {gau }}$ takes into account the unfiltered Gaussian noise, and finally $\mathrm{NCD}_{\text {dis }}$ measures the unwanted distortion produced by the filtering. Since impulse noise generally affects only a subset of the image pixels, we can easily define the $\mathrm{NCD}_{\text {imp }}$ component using relation (1), where $D$ represents in this case the subset $C_{1}$ of coordinates where noise pulses occurred $\left(D \equiv C_{1}\right)$.

The $\mathrm{NCD}_{\text {gau }}$ and $\mathrm{NCD}_{\text {dis }}$ must be evaluated in the subset $\mathrm{C}_{2}$ of pixel coordinates where impulse noise has not been superimposed. Their definition is more difficult because it is expected that almost every pixel in $C_{2}$ is degraded by Gaussian noise. In this case, a possible choice for measuring the noise cancellation and the edge preservation could be to focus on the uniform and nonuniform areas, according to visual perception. A more effective solution for separating the evaluation of distortion from that of the residual noise consists, however, in determining the distortion area, that is, the pixel regions where the filter (adopting the same param eter settings) would blur the original noise-free image. In principle, a map of the differences between the original and the blurred picture would suffice for perfectly noiseless synthetic test images. In practice, most test pictures used in the literature contain small amounts of noise. Thus, a soft threshold approach is needed. For the computation of such distortion area, the RGB color space suffices. Let $\mathbf{r}(\mathbf{c})=$ $\left[r_{R}(\mathbf{c}), r_{G}(\mathbf{c}), r_{B}(\mathbf{c})\right]^{T}$ be the vector (in the RGB color coordinate system) representing the pixel at spatial position $\mathbf{c}=\left[c_{1}, c_{2}\right]$ in the original noise-free image and let $\mathbf{s}(\mathbf{c})=$ $\left[s_{R}(\mathbf{c}), s_{G}(\mathbf{c}), s_{B}(\mathbf{c})\right]^{T}$ be the corresponding pixel in the blurred picture that is produced when the original noise-free image is filtered (adopting the same parameter settings). Let $\delta(\mathbf{c})$ represent the dissimilarity (or distance) between $s(\mathbf{c})$ and $r(\mathbf{c})$ :

\section{$\delta(\mathbf{c})$}

$$
=\sqrt{\left(s_{R}(\mathbf{c})-r_{R}(\mathbf{c})\right)^{2}+\left(s_{G}(\mathbf{c})-r_{G}(\mathbf{c})\right)^{2}+\left(s_{B}(\mathbf{c})-r_{B}(\mathbf{c})\right)^{2}} .
$$

Thus, we shall define the $\mathrm{NCD}_{\text {gau }}$ and $\mathrm{NCD}_{\text {dis }}$ as follows:

$$
\begin{aligned}
\mathrm{NCD}_{\text {dis }} & =\frac{\sum_{\mathbf{c} \in C_{2}} \beta(\mathbf{c}) \Delta E(\mathbf{c})}{\sum_{\mathbf{c} \in C} \sqrt{\left(\bar{r}_{L}(\mathbf{c})\right)^{2}+\left(\bar{r}_{u}(\mathbf{c})\right)^{2}+\left(\bar{r}_{v}(\mathbf{c})\right)^{2}}}, \\
\mathrm{NCD}_{\text {gau }} & =\frac{\sum_{\mathbf{c} \in C_{2}}[1-\beta(\mathbf{c})] \Delta E(\mathbf{c})}{\sum_{\mathbf{c} \in C} \sqrt{\left(\bar{r}_{L}(\mathbf{c})\right)^{2}+\left(\bar{r}_{u}(\mathbf{c})\right)^{2}+\left(\bar{r}_{v}(\mathbf{c})\right)^{2}}},
\end{aligned}
$$

where $\Delta E(\mathbf{c})$ is the color difference (or error) evaluated in the Luv perceptually uniform color space:

$$
\begin{gathered}
\Delta E(\mathbf{c})=\left(\left(\bar{y}_{L}(\mathbf{c})-\bar{r}_{L}(\mathbf{c})\right)^{2}+\left(\bar{y}_{u}(\mathbf{c})-\bar{r}_{u}(\mathbf{c})\right)^{2}\right. \\
\left.+\left(\bar{y}_{v}(\mathbf{c})-\bar{r}_{v}(\mathbf{c})\right)^{2}\right)^{1 / 2}
\end{gathered}
$$

and $\beta(\mathbf{c})$ denotes the degree of blur at location $\mathbf{c}=\left[\mathrm{c}_{1}, \mathrm{c}_{2}\right]$. We evaluate this degree by means of the parameterized function depicted in Figure 1, where $a$ and $b$ are parameters.

Clearly, the $\mathrm{NCD}_{\text {dis }}$ mainly collects the color errors in the distortion area $(\beta=1)$, whereas the $\mathrm{NCD}_{\text {gau }}$ mainly deals with the errors that are located in the uniform regions $(\beta=$ $0)$. For synthetic test images (including perfectly uniform regions), a crisp threshold is the natural choice ( $a=b$, 


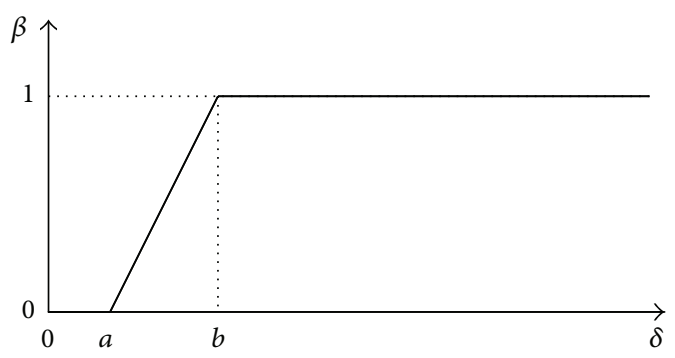

FIgURE 1: Graphical representation of the function $\beta$.

$a \approx 0$ ). For test images represented by real pictures having nonzero noise variance in the background, a soft threshold $(a>0, b>a)$ is a more suitable choice. Since the noise variance should be small (typically $\sigma^{2} \leq 4$ ), small values for parameters $a$ and $b$ are appropriate; for example, $a=4$ and $b=20$. This choice is based on a heuristic approach (too small values could wrongly extend the distortion area and then yield an excess of $\mathrm{NCD}_{\text {dis }}$, whereas too large values would produce the opposite effect). Notice that, in the proposed approach, once $a$ and $b$ have been chosen, the extension of the distortion area depends upon the kind of filter and the window size, as it will be shown in the next section. It should be observed that if impulse noise is the only kind of noise degrading the image, the NCD is decomposed into two components only: the residual noise component $\mathrm{NCD}_{\text {imp }}$ and a distortion component $\mathrm{NCD}_{\text {dis }}$ that, in this case, can be evaluated by the difference NCD - NCD ${ }_{\text {imp }}$. Similarly, if the picture is corrupted by Gaussian noise only, the NCD is again decomposed into two components $\mathrm{NCD}_{\text {gau }}$ and $\mathrm{NCD}_{\text {dis }}$ only. As mentioned in Section 1, the NCD computes all the color errors in a perceptually uniform color space in order to evaluate the perceptual closeness of a filtered image to the uncorrupted original. Clearly, the NCD by itself could not distinguish between errors caused by filtering distortion or by insufficient noise cancellation. Splitting the NCD into appropriate components, such as the $\mathrm{NCD}_{\text {imp }}, \mathrm{NCD}_{\text {gau }}$, and $\mathrm{NCD}_{\text {dis }}$, removes this limitation and retains the specific advantage of the NCD approach.

\section{How the Method Works}

In order to analyze the behavior of the proposed method, we generated the test image shown in Figure 2(a). In this picture, perfectly uniform regions and image edges are located into different areas. We produced a noisy picture by adding zeromean Gaussian noise (with standard deviation $\sigma=30$ ) into some uniform regions only (Figure 2(b)). In this experiment we resorted to the vector mean filter because its behavior is well known. We processed the noisy picture by adopting $(2 N+1) \times(2 N+1)$ filters with increasing window size $(N=2,3,4,5)$. The results of the processing are depicted in Figure 3. From visual inspection, we can easily see that the noise is reduced at the price of an increasing detail blur (left to right). It is expected that measures of residual noise and unwanted blur should yield numerical evaluations that comply with these observations.
TABLE 1: List of NCD, $\mathrm{NCD}_{\text {gau }}$, and $\mathrm{NCD}_{\text {dis }}$ evaluations for $(2 \mathrm{~N}+1) \times$ $(2 N+1)$ vector mean filters.

\begin{tabular}{cccc}
\hline$N$ & $\mathrm{NCD}\left(\times 10^{2}\right)$ & $\mathrm{NCD}_{\text {gau }}\left(\times 10^{2}\right)$ & $\mathrm{NCD}_{\text {dis }}\left(\times 10^{2}\right)$ \\
\hline 2 & 10.92 & 2.72 & 8.21 \\
3 & 13.15 & 1.96 & 11.19 \\
4 & 14.73 & 1.55 & 13.18 \\
5 & 16.05 & 1.29 & 14.75 \\
\hline
\end{tabular}

The distortion areas computed in our method are shown in Figure 4, where black and white, respectively, denote $\beta=0$ and $\beta=1$. To evaluate these areas we chose a crisp threshold ( $a=b=0$ ), so that $\beta$ became a two-valued quantity revealing distortion $(\delta>0 \Rightarrow \beta=1$ ) or no distortion $(\delta=0 \Rightarrow \beta=0)$. The extension of such areas increases as the window size increases, as it should be. The results given by our method are listed in Table 1 . We can observe that the proposed NCD decomposition is in perfect agreement with the filtering behavior. The values of the $\mathrm{NCD}_{\text {gau }}$ (third column) decrease as the noise cancellation becomes stronger. Similarly, larger values of the $\mathrm{NCD}_{\text {dis }}$ highlight the growing blur in the filtered images.

For real images used as test pictures, a soft threshold approach is required, as mentioned in the previous section. In this case, in order to improve the accuracy of the $\mathrm{NCD}_{\text {gau }}$ and $\mathrm{NCD}_{\text {dis }}$ evaluations, we also adopt a simple calibration procedure that subtracts from $\mathrm{NCD}_{\text {gau }}$ (and adds to $\mathrm{NCD}_{\text {dis }}$ ) the possibly nonzero $\mathrm{NCD}_{\text {gau }}^{*}$ component measured in the uncorrupted image.

\section{Results of Computer Simulations}

In order to evaluate the performance of the proposed technique, we report and discuss in this section the results of many computer simulations based on images of the wellknown Kodak test set [26]. In the following experiments four pictures from this set are considered. They are depicted in Figure 5. All of these images are 24-bit color pictures whose size is 512-by-512 pixels.

In this first experiment we briefly highlight the advantages of our method over the classical MSE and MAE evaluations (an in-depth analysis of the inaccuracy of MAE and MSE is reported in [21]. We generated two images having very different combinations of residual noise and edge preservation, as in [21]. We adopted vector median filters having different window sizes to produce these results. Figure 6(a) shows the input noisy picture where each channel component is degraded by fixed valued impulse noise with probability $p \approx 40 \%$. The filtered images are shown in Figure 6(b) (smaller window yielding more residual noise and less blur) and in Figure 6(c) (larger window giving less residual noise and more blur). The differences in terms of detail preservation and noise removal are apparent from visual inspection. However, they cannot be distinguished in terms of MSE and MAE (see Table 2). 


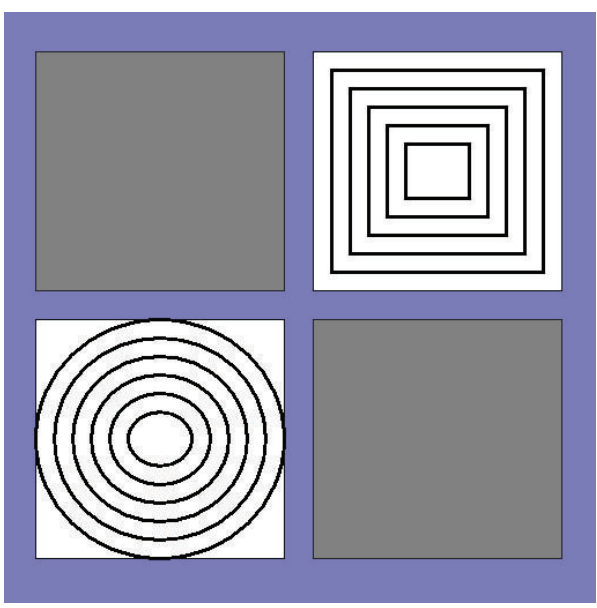

(a)

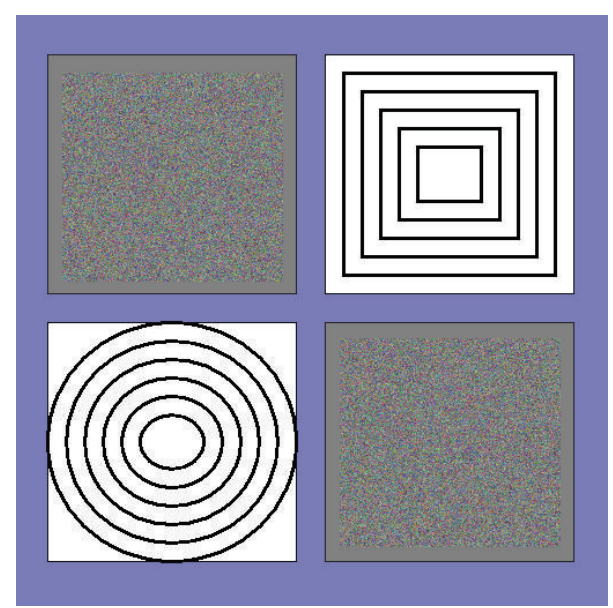

(b)

FIGURE 2: Test images: (a) noise-free and (b) corrupted by Gaussian noise.

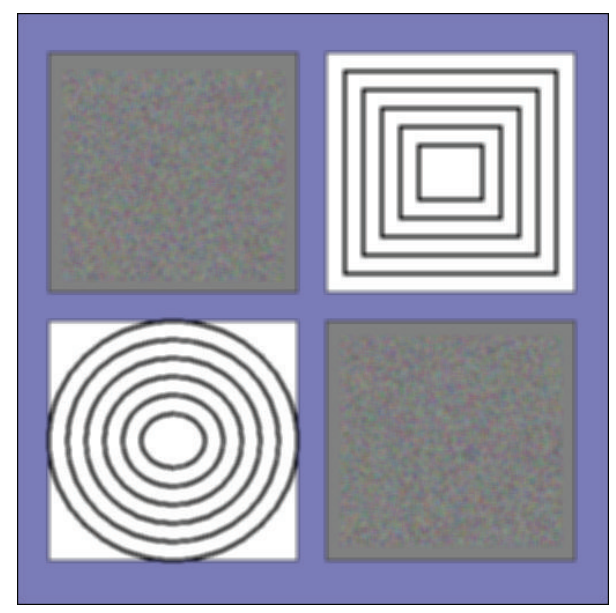

(a)

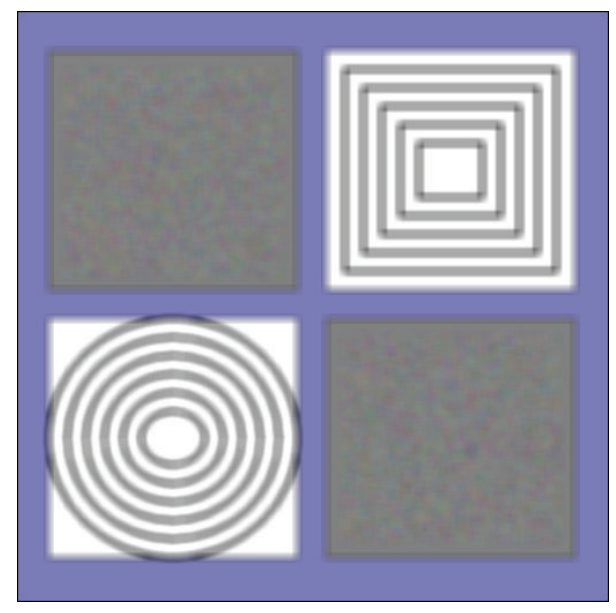

(c)

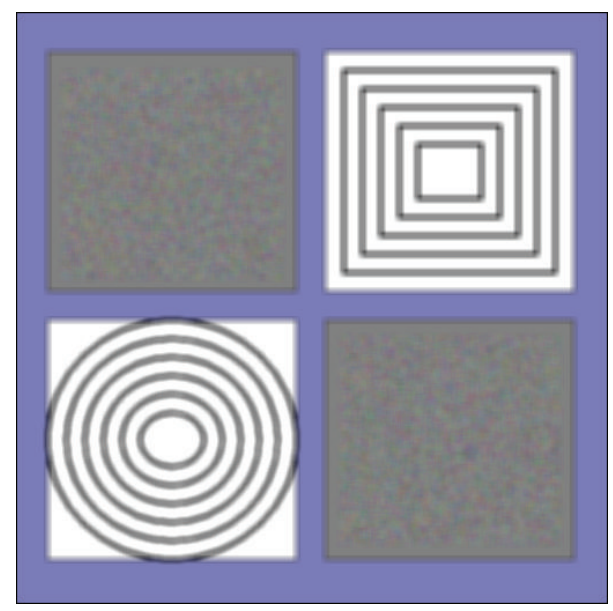

(b)

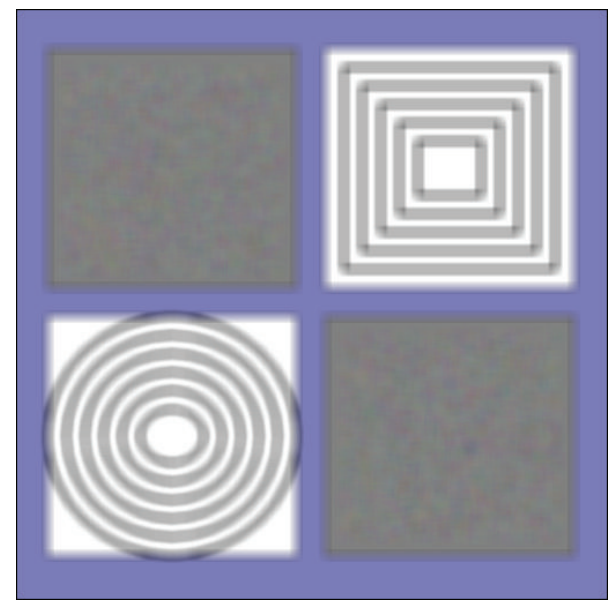

(d)

Figure 3: Results of $(2 N+1) \times(2 N+1)$ filtering: (a) $N=2$, (b) $N=3$, (c) $N=4$, and (d) $N=5$. 


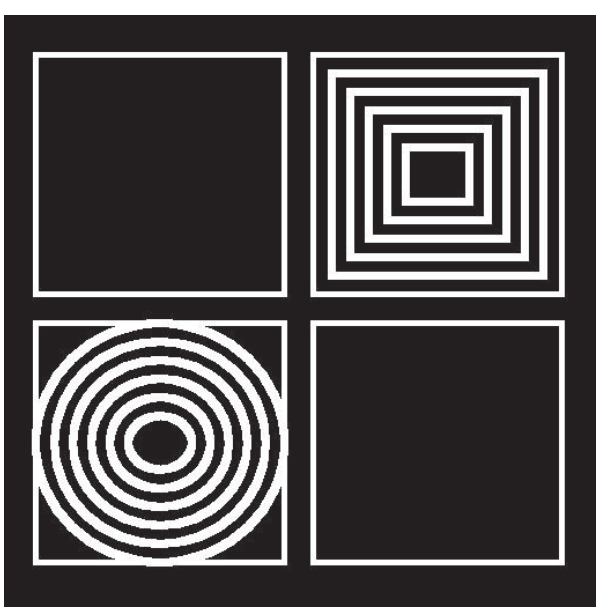

(a)

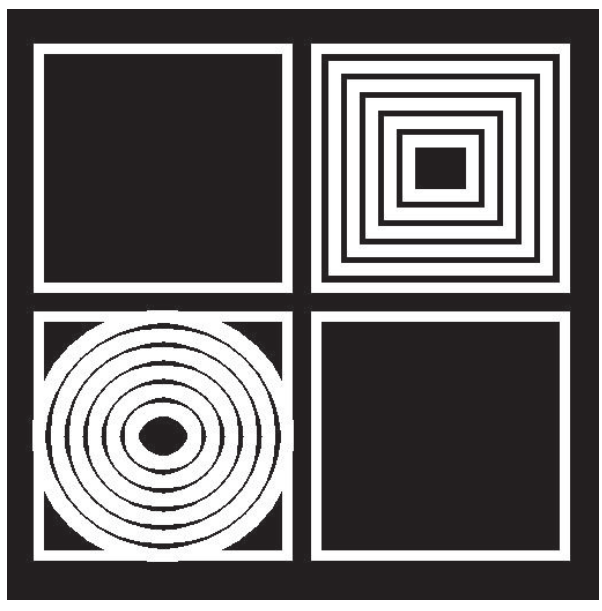

(c)

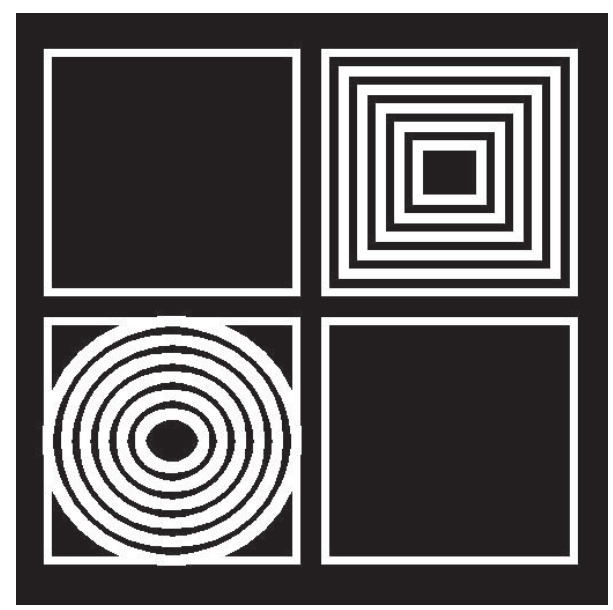

(b)

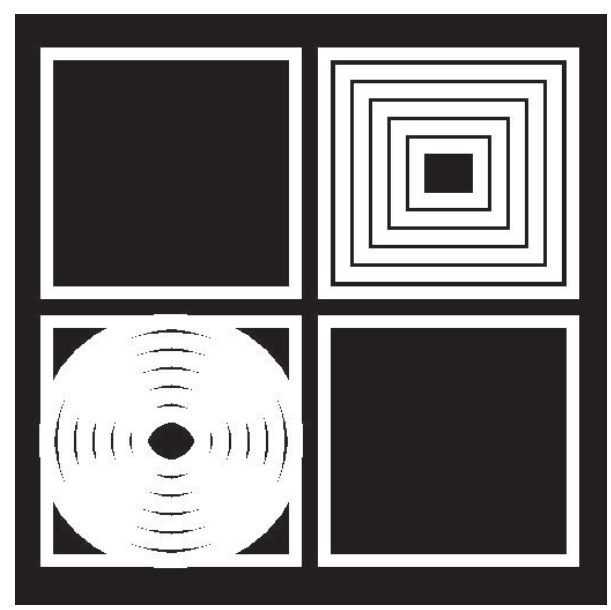

(d)

Figure 4: Distortion areas for $(2 N+1) \times(2 N+1)$ windows: (a) $N=2$, (b) $N=3$, (c) $N=4$, and (d) $N=5$.

TABLE 2: Advantages of our method over the classical MSE and MAE evaluations ("Parrots" image corrupted by fixed-value impulse noise and filtered by 5 -point and $5 \times 5$ vector median operators).

\begin{tabular}{lcccc}
\hline Image & $\mathrm{MSE}$ & $\mathrm{MAE}$ & $\mathrm{NCD}_{\text {imp }}\left(\times 10^{2}\right)$ & $\mathrm{NCD}_{\text {dis }}\left(\times 10^{2}\right)$ \\
\hline Figure 6(b) & 77 & 3.6 & 3.37 & 1.42 \\
Figure 6(c) & 77 & 3.6 & 1.58 & 2.08 \\
\hline
\end{tabular}

Conversely, our method correctly characterizes the mentioned filtering behavior. The $\mathrm{NCD}_{\text {imp }}$ can measure the different amounts of residual noise, whereas the MSE cannot (the filtered images have the same MSE). The $\mathrm{NCD}_{\text {dis }}$ can focus on the image distortion only, whereas the MAE cannot (the filtered images have the same MAE).

In the second experiment we considered an example of image quality index that follows human perception: the color quality index CQI [27]. As in the previous case, we generated two images with different combinations of unfiltered noise and collateral blur. The input picture, corrupted by Gaussian noise $(\sigma=22)$, is reported in Figure 7(a). We adopted vector mean filters having different window sizes to produce
TABLE 3: List of CQI, $\mathrm{NCD}_{\text {gau }}$, and $\mathrm{NCD}_{\text {dis }}$ evaluations ("Flowers" image corrupted by Gaussian noise and filtered by $3 \times 3$ and $5 \times 5$ vector mean operators).

\begin{tabular}{lccc}
\hline Image & $\mathrm{CQI}$ & $\mathrm{NCD}_{\text {gau }}\left(\times 10^{2}\right)$ & $\mathrm{NCD}_{\text {dis }}\left(\times 10^{2}\right)$ \\
\hline Figure 7(b) & 0.744 & 11.04 & 4.90 \\
Figure 7(c) & 0.744 & 4.91 & 7.07 \\
\hline
\end{tabular}

the results in Figure 7(b) (more residual noise, less blur) and Figure 7(c) (less residual noise, more blur). The list of quantitative evaluations is reported in Table 3. The same score is obtained (CQI = 0.744) for both images, because different mixtures of residual noise and distortion yield the same loss of perceived image quality. Again, our method can easily separate (and measure) such effects.

In the third experiment we considered for a comparison our previous vector technique operating in the YIQ color space [25]. In this approach, the MSE evaluated in the luminance channel is split into two components $\mathrm{MSE}_{\mathrm{A}}$ and $\mathrm{MSE}_{\mathrm{B}}$ that, respectively, estimate the noise cancellation and the detail preservation. We chose the "House" picture as test 


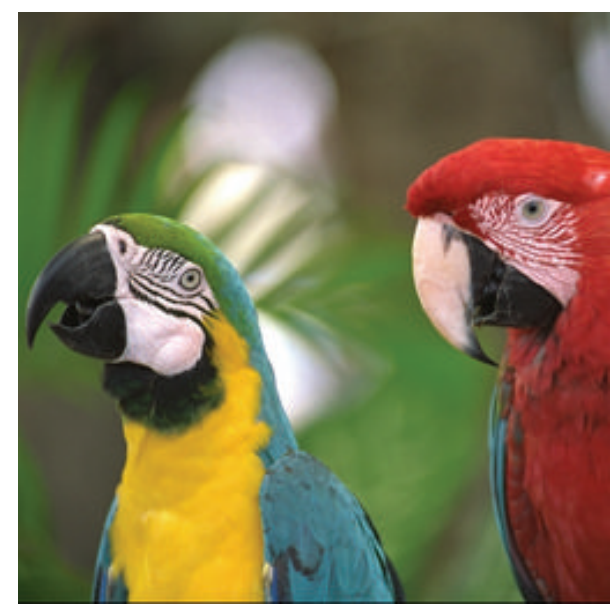

(a)

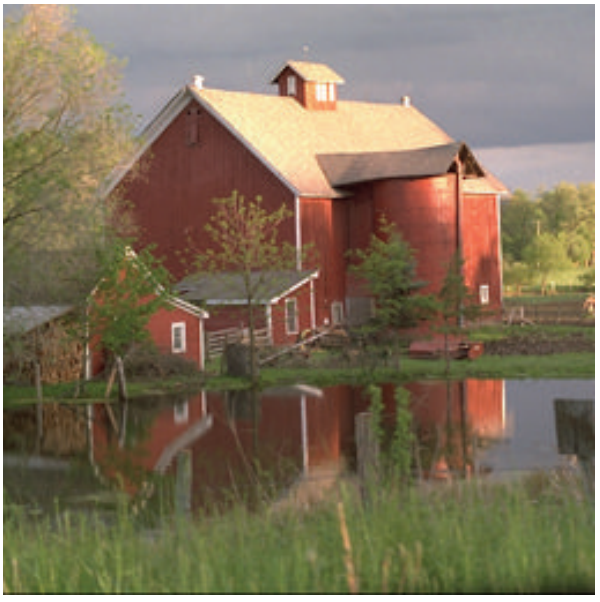

(c)

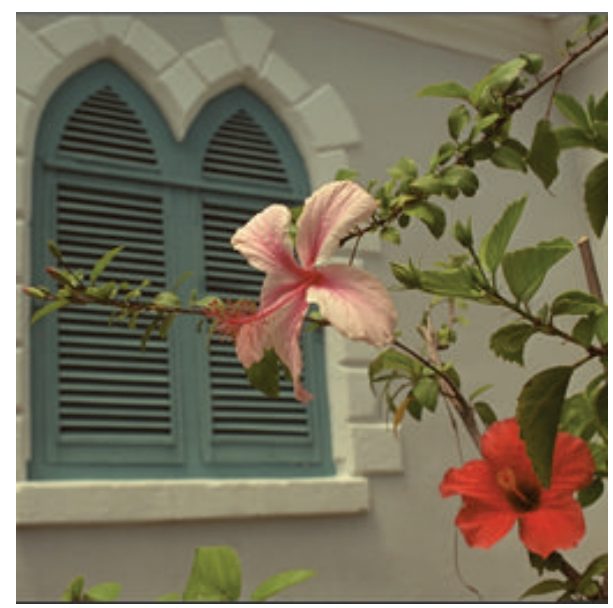

(b)

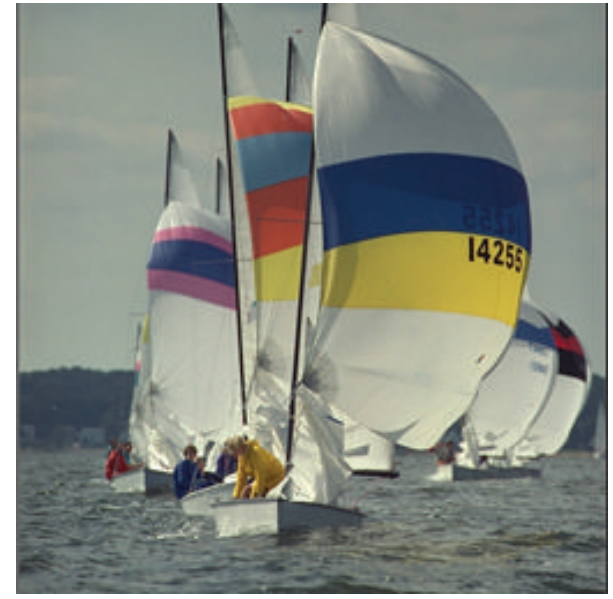

(d)

Figure 5: Test images used in the experiments: (a) Parrots, (b) Flowers, (c) House, and (d) Boat.

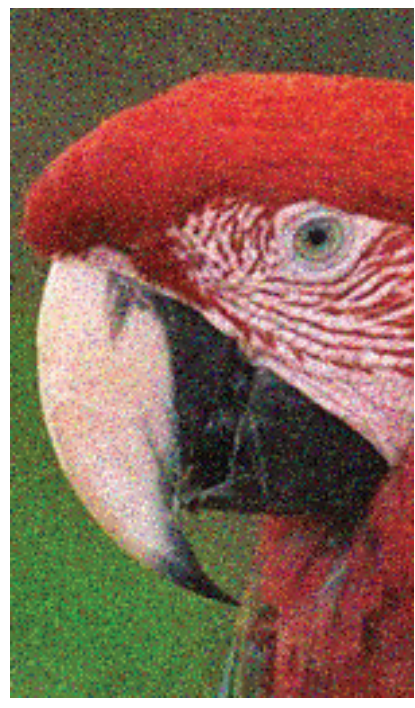

(a)

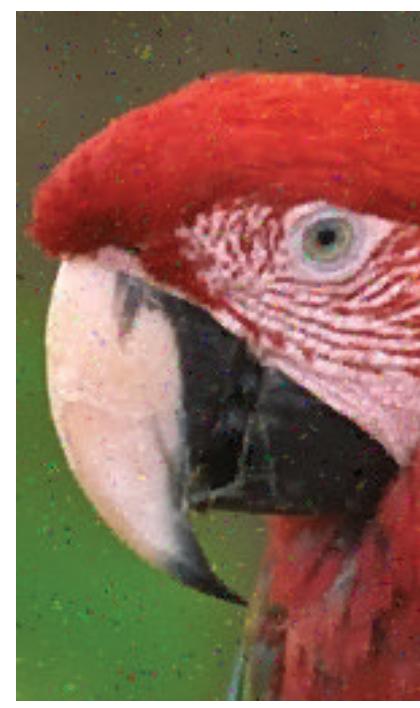

(b)

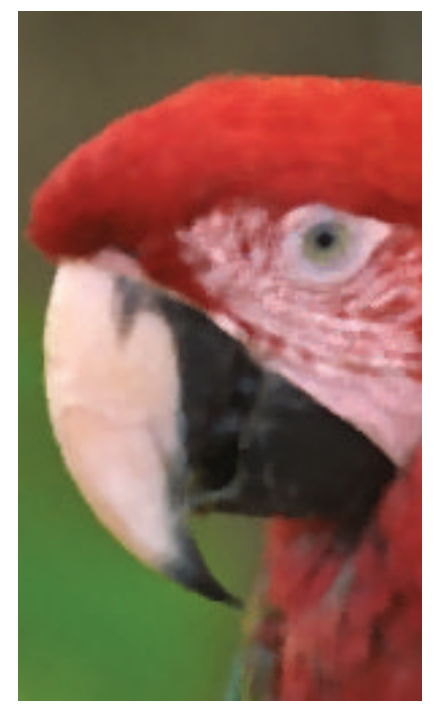

(c)

FIgURE 6: (a) Noisy data, (b) result yielded by the 5 -point vector median, and (c) result yielded by the $5 \times 5$ vector median. 


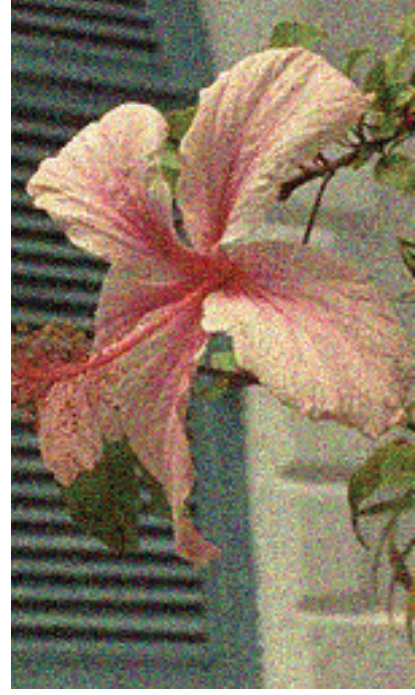

(a)

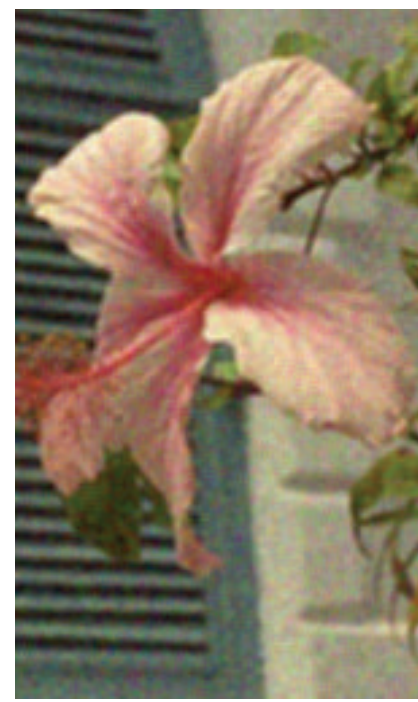

(b)

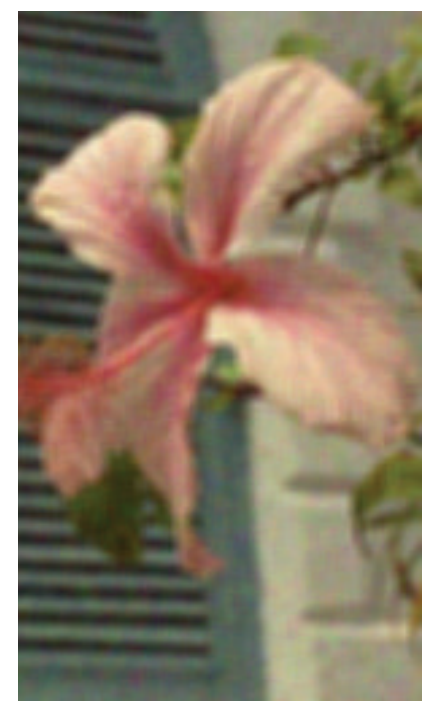

(c)

Figure 7: (a) Noisy data, (b) result yielded by the $3 \times 3$ vector mean filter, and (c) result yielded by the $5 \times 5$ vector mean filter.

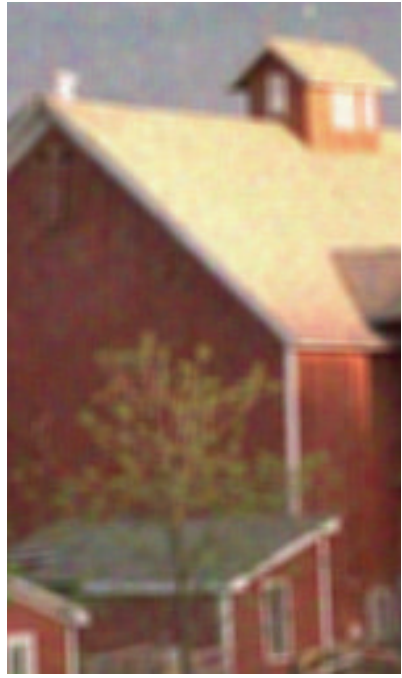

(a)

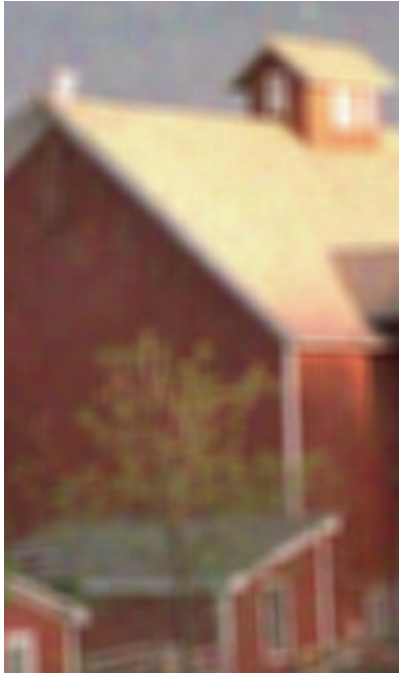

(b)

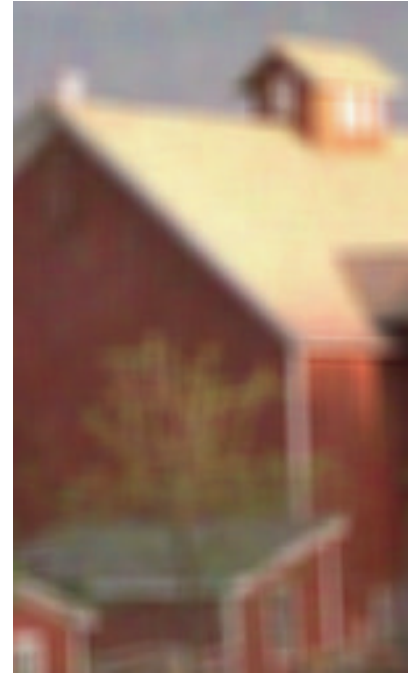

(c)

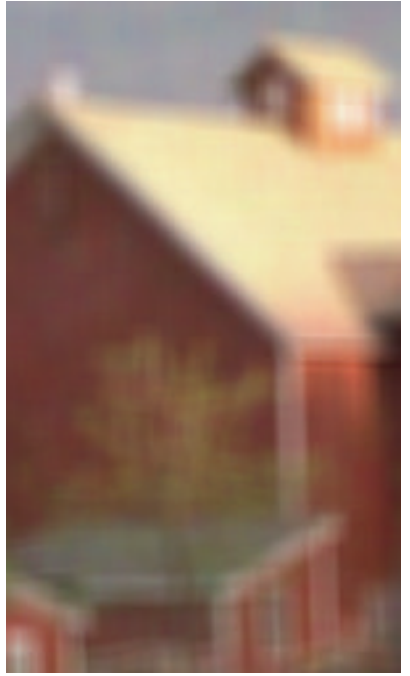

(d)

Figure 8: Results of $(2 N+1) \times(2 N+1)$ vector mean filtering: (a) $N=2$, (b) $N=3$, (c) $N=4$, and (d) $N=5$.

image and we generated a noisy version of it by adding zeromean Gaussian noise with $\sigma=20$.

As done in Section 3, we adopted the well-known vector mean filter and we processed the noisy data by adopting $(2 N+1) \times(2 N+1)$ operators with increasing window size $(N=2,3,4,5)$. The results are reported in Table 4 . Portions of the processed images are shown in Figure 8. It can be seen that the residual noise decreases as the window becomes larger. Hence, it is expected that the corresponding filtering errors decrease. However, this does not occur for the $\mathrm{MSE}_{\mathrm{A}}$ : growing values of the $\mathrm{MSE}_{\mathrm{A}}$ characterize images filtered with larger windows (second column in Table 4). The proposed $\mathrm{NCD}_{\text {gau }}$ is much more accurate (fourth column). Its values become smaller as the smoothing effect increases, as it should be.
TABLE 4: List of $\mathrm{MSE}_{\mathrm{A}}, \mathrm{MSE}_{\mathrm{B}}, \mathrm{NCD}_{\text {gau }}$, and $\mathrm{NCD}_{\text {dis }}$ values ("House" image corrupted by Gaussian noise with $\sigma=20$ and filtered by $(2 N+$ $1) \times(2 N+1)$ vector mean operators $)$.

\begin{tabular}{ccccc}
\hline$N$ & $\mathrm{MSE}_{\mathrm{A}}$ & $\mathrm{MSE}_{\mathrm{B}}$ & $\mathrm{NCD}_{\text {gau }}\left(\times 10^{2}\right)$ & $\mathrm{NCD}_{\text {dis }}\left(\times 10^{2}\right)$ \\
\hline 2 & 23.94 & 30.74 & 3.42 & 8.19 \\
3 & 30.19 & 37.49 & 1.88 & 9.25 \\
4 & 34.93 & 41.82 & 1.17 & 10.20 \\
5 & 38.97 & 45.07 & 0.79 & 11.04 \\
\hline
\end{tabular}

The same behavior can be observed if we adopt different test pictures, such as the "Boat" picture (Table 5). 


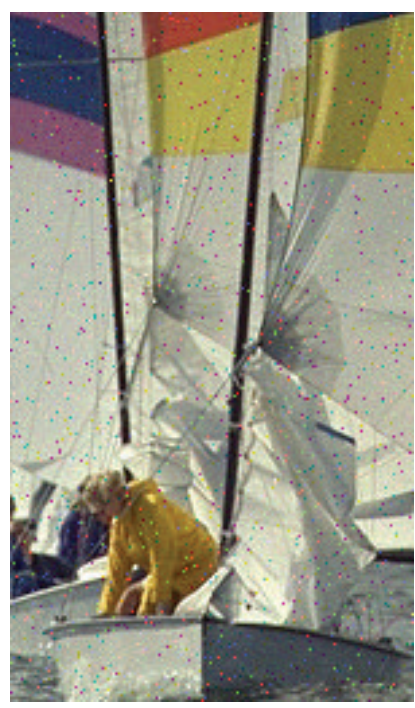

(a)

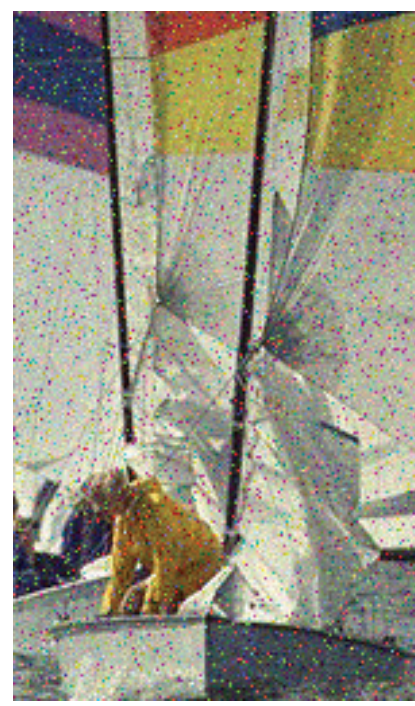

(b)

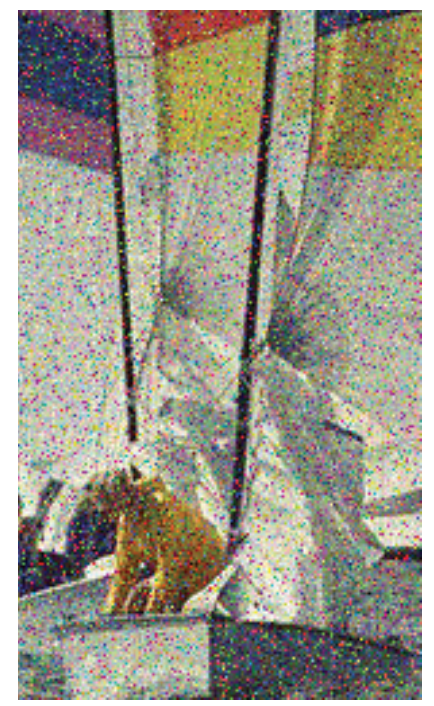

(c)

Figure 9: Test images corrupted by different amounts of Gaussian and impulse noise: (a) $\sigma=5, p=5 \%$; (b) $\sigma=15, p=15 \%$; (c) $\sigma=25$, and $p=25 \%$.

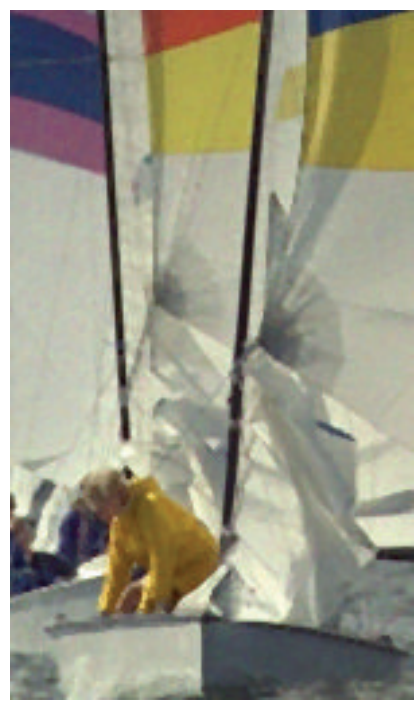

(a)

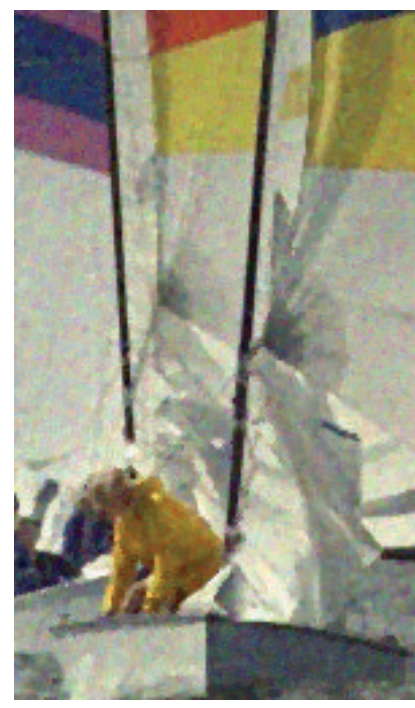

(b)

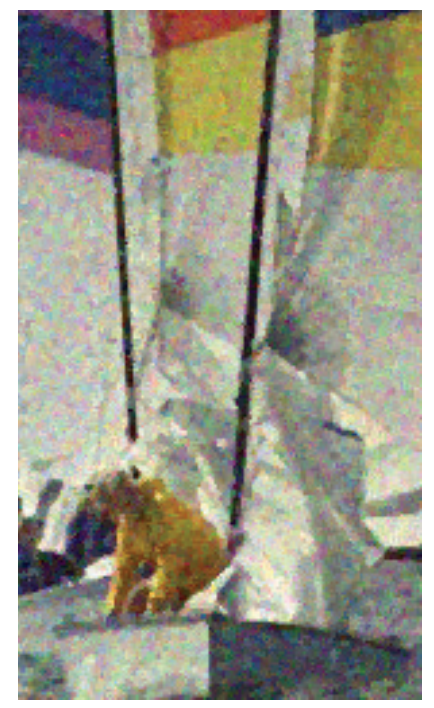

(c)

FIGURE 10: Corresponding results yielded by the FPGA filter.

TABLE 5: List of $\mathrm{MSE}_{\mathrm{A}}, \mathrm{MSE}_{\mathrm{B}}, \mathrm{NCD}_{\text {gau }}$, and $\mathrm{NCD}_{\text {dis }}$ values (“Boat" image corrupted by Gaussian noise with $\sigma=20$ and filtered by $(2 \mathrm{~N}+$ $1) \times(2 N+1)$ vector mean operators $)$.

\begin{tabular}{ccccc}
\hline$N$ & $\mathrm{MSE}_{\mathrm{A}}$ & $\mathrm{MSE}_{\mathrm{B}}$ & $\mathrm{NCD}_{\text {gau }}\left(\times 10^{2}\right)$ & $\mathrm{NCD}_{\text {dis }}\left(\times 10^{2}\right)$ \\
\hline 2 & 21.59 & 32.31 & 3.83 & 6.44 \\
3 & 32.16 & 45.20 & 2.24 & 7.37 \\
4 & 41.79 & 54.94 & 1.44 & 8.20 \\
5 & 50.34 & 62.14 & 1.00 & 8.94 \\
\hline
\end{tabular}

The different performance of the previous and new method mainly resides in the different error decomposition schemes. The previous method focused on the uniform and nonuniform areas of a picture to distinguish between noise cancellation and detail preservation, according to visual perception. Here, the $\mathrm{MSE}_{\mathrm{A}}$ and $\mathrm{MSE}_{\mathrm{B}}$ components are simply obtained by resorting to an edge map given by the Sobel operator. However, since this map does not depend upon the actual filtering action, errors due to blur (possibly located in the exterior of this map) can wrongly be added to the $\mathrm{MSE}_{\mathrm{A}}$, thus increasing its value. The decomposition scheme adopted in the proposed method, on the contrary, is based on a distortion area that depends upon the actual filtering: it can increase when the smoothing is stronger and generates more blur. 


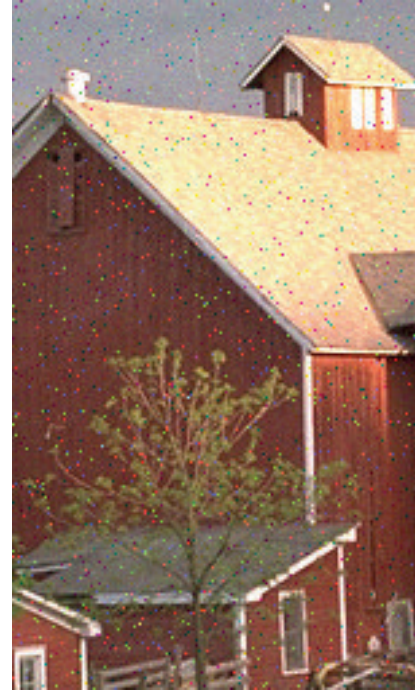

(a)

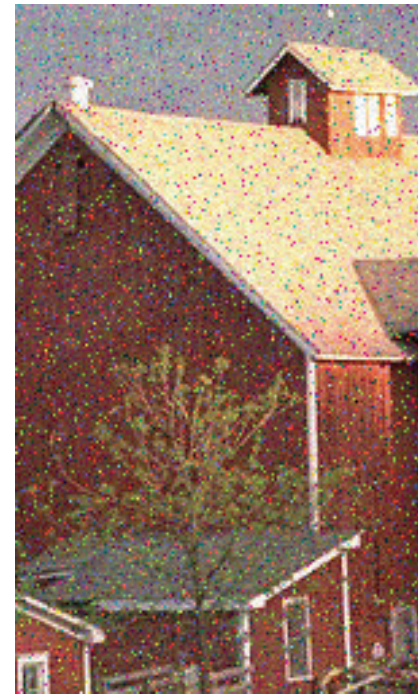

(b)

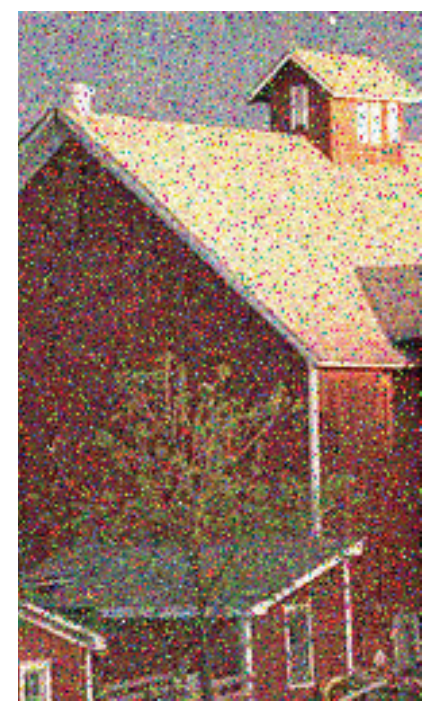

(c)

Figure 11: Test images corrupted by different amounts of Gaussian and impulse noise: (a) $\sigma=5, p=5 \%$; (b) $\sigma=15, p=15 \%$; (c) $\sigma=25$, and $p=25 \%$.

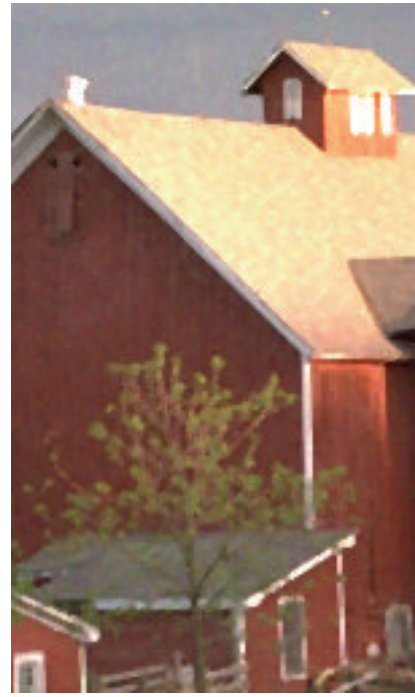

(a)

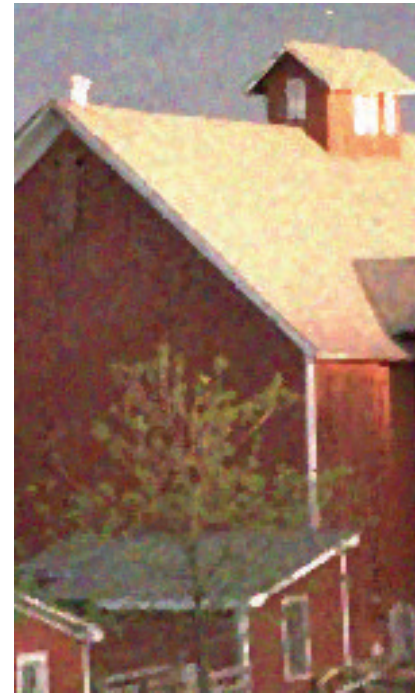

(b)

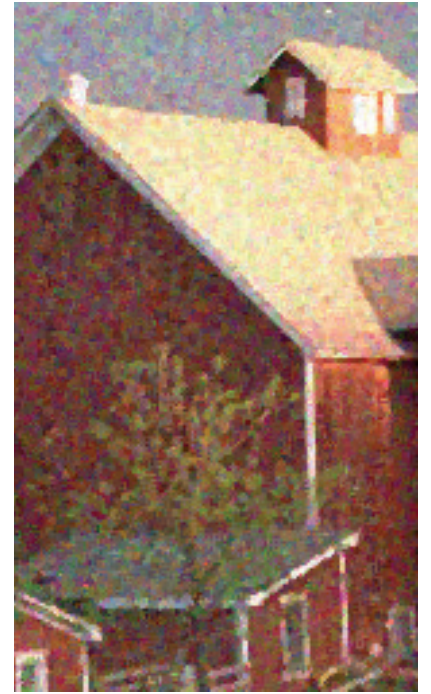

(c)

FIGURE 12: Corresponding results yielded by the FPGA filter.

Thus, even in the presence of large window filters, errors caused by blur are correctly included into the corresponding $\mathrm{NCD}_{\text {dis }}$ component. Consequently, the $\mathrm{NCD}_{\text {gau }}$ can correctly take into account the residual noise only.

In the fourth experiment we finally considered the case of mixed noise. We chose the "Boat" image and we generated three noisy pictures by adding growing amounts of Gaussian and impulse noise (Figure 9). We filtered the noisy pictures by using the FPGA filter [14] because it is known that this operator is very effective in removing mixed noise from color images. We set the filtering parameters according to the values of the standard deviation $\sigma$ (Gaussian noise) and probability $p$ (impulse noise), as suggested in [14]. The filtered pictures are shown in Figure 10 for visual inspection. The corresponding evaluations given by the new technique and the previous one are listed in Table 6. The different amounts of residual impulse and Gaussian noise are, respectively, measured by the $\mathrm{NCD}_{\text {imp }}$ and $\mathrm{NCD}_{\text {gau }}$ components (proposed method). Clearly, the sole $\mathrm{MSE}_{\mathrm{A}}$ cannot separate these features (second column), like all other existing metrics in the literature. Similar results can be obtained if we adopt different pictures, such as the "House" image (Figure 11). 
TABLE 6: List of $\mathrm{MSE}_{\mathrm{A}}, \mathrm{MSE}_{\mathrm{B}}, \mathrm{NCD}_{\mathrm{imp}}, \mathrm{NCD}_{\text {gau }}$, and $\mathrm{NCD}_{\text {dis }}$ values ("Boat" image corrupted by growing amounts of mixed noise and filtered by the FPGA operator).

\begin{tabular}{|c|c|c|c|c|c|}
\hline Image & $\mathrm{MSE}_{\mathrm{A}}$ & $\mathrm{MSE}_{\mathrm{B}}$ & $\mathrm{NCD}_{\text {imp }}\left(\times 10^{2}\right)$ & $\mathrm{NCD}_{\text {gau }}\left(\times 10^{2}\right)$ & $\mathrm{NCD}_{\text {dis }}\left(\times 10^{2}\right)$ \\
\hline Figure 10(a) & 8.43 & 7.60 & 0.39 & 3.87 & 2.90 \\
\hline Figure 10(b) & 18.18 & 12.48 & 2.79 & 11.64 & 3.73 \\
\hline Figure $10(c)$ & 38.46 & 20.62 & 7.36 & 18.25 & 4.53 \\
\hline
\end{tabular}

TABLE 7: List of $\mathrm{MSE}_{\mathrm{A}}, \mathrm{MSE}_{\mathrm{B}}, \mathrm{NCD}_{\text {imp }}, \mathrm{NCD}_{\text {gau }}$, and $\mathrm{NCD}_{\text {dis }}$ values ("House" image corrupted by growing amounts of mixed noise and filtered by the FPGA operator).

\begin{tabular}{lccccc}
\hline Image & $\mathrm{MSE}_{\mathrm{A}}$ & $\mathrm{MSE}_{\mathrm{B}}$ & $\mathrm{NCD}_{\text {imp }}\left(\times 10^{2}\right)$ & $\mathrm{NCD}_{\text {gau }}\left(\times 10^{2}\right)$ & 3.67 \\
Figure 12(a) & 12.03 & 10.74 & 0.45 & 11.02 & 4.38 \\
Figure 12(b) & 21.67 & 15.17 & 2.96 & 17.35 & 5.52 \\
Figure 12(c) & 41.43 & 22.39 & 7.66 & $\mathrm{NCD}_{\text {dis }}\left(\times 10^{2}\right)$ \\
\hline
\end{tabular}

The filtered data are shown in Figure 12 for visual inspection. The corresponding evaluations given by the new method and the previous one are listed in Table 7.

\section{Conclusions}

Performance evaluation of noise reduction techniques needs appropriate full-reference metrics able to measure the different amounts of residual noise and filtering distortion. In this paper we have presented a new method for evaluating such features in color images restored from impulse and Gaussian noise. The approach is based on the decomposition of the NCD into three components that, respectively, measure the ability of a filter to remove noise pulses $\left(\mathrm{NCD}_{\mathrm{imp}}\right)$, to reduce Gaussian noise $\left(\mathrm{NCD}_{\text {gau }}\right)$, and to preserve the image details $\left(\mathrm{NCD}_{\text {dis }}\right)$. These new measures retain the specific advantage of the NCD, that is, the evaluation of color errors in a perceptually uniform color space. On the other hand, they overcome the limitation of the sole NCD that cannot distinguish between color errors due to filtering distortion and insufficient noise cancellation.

Results of computer simulations dealing with different pictures corrupted by impulse and Gaussian noise have shown that the proposed method outperforms classical and vector metrics in the literature in the evaluation of the different amounts of residual noise and distortion given by a denoising filter.

\section{Conflict of Interests}

The author declares that there is no conflict of interests regarding the publication of this paper.

\section{References}

[1] K. N. Plataniotis and A. N. Venetsanopoulos, Color Image Processing and Application, Springer, New York, NY, USA, 2000.

[2] R. Lukac, B. Smolka, K. Martin, K. N. Plataniotis, and A. N. Venetsanopoulos, "Vector filtering for color imaging," IEEE Signal Processing Magazine, vol. 22, no. 1, pp. 74-86, 2005.

[3] R. Lukac and K. N. Plataniotis, "A taxonomy of color image filtering and enhancement solutions," in Advances in Imaging and
Electron Physics, W. Hawkes, Ed., vol. 140, pp. 187-264, Elsevier, New York, NY, USA, 2006.

[4] R. Lukac, B. Smolka, K. N. Plataniotis, and A. N. Venetsanopoulos, "Selection weighted vector directional filters," Computer Vision and Image Understanding, vol. 94, no. 1-3, pp. 140-167, 2004.

[5] R. Lukac, B. Smolka, K. N. Plataniotis, and A. N. Venetsanopoulos, "Vector sigma filters for noise detection and removal in color images," Journal of Visual Communication and Image Representation, vol. 17, no. 1, pp. 1-26, 2006.

[6] Y. Li, G. R. Arce, and J. Bacca, "Weighted median filters for multichannel signals," IEEE Transactions on Signal Processing, vol. 54, no. 11, pp. 4271-4281, 2006.

[7] S. Schulte, V. De Witte, M. Nachtegael, D. van der Weken, and E. E. Kerre, "Fuzzy two-step filter for impulse noise reduction from color images," IEEE Transactions on Image Processing, vol. 15, no. 11, pp. 3567-3578, 2006.

[8] S. Schulte, V. De Witte, and E. E. Kerre, "A fuzzy noise reduction method for color images," IEEE Transactions on Image Processing, vol. 16, no. 5, pp. 1425-1436, 2007.

[9] S. Schulte, S. Morillas, V. Gregori, and E. E. Kerre, "A new fuzzy color correlated impulse noise reduction method," IEEE Transactions on Image Processing, vol. 16, no. 10, pp. 2565-2575, 2007.

[10] P.-E. Ng and K.-K. Ma, "A switching median filter with boundary discriminative noise detection for extremely corrupted images," IEEE Transactions on Image Processing, vol. 15, no. 6, pp. 1506-1516, 2006.

[11] Y. Dong and S. Xu, "A new directional weighted median filter for removal of random-valued impulse noise," IEEE Signal Processing Letters, vol. 14, no. 3, pp. 193-196, 2007.

[12] Y. Li, F.-L. Chung, and S. Wang, "A robust neuro-fuzzy network approach to impulse noise filtering for color images," Applied Soft Computing Journal, vol. 8, no. 2, pp. 872-884, 2008.

[13] Z. Xu, H. R. Wu, B. Qiu, and X. Yu, "Geometric features-based filtering for suppression of impulse noise in color images," IEEE Transactions on Image Processing, vol. 18, no. 8, pp. 1742-1759, 2009.

[14] S. Morillas, V. Gregori, and A. Hervás, "Fuzzy peer groups for reducing mixed Gaussian-impulse noise from color images," IEEE Transactions on Image Processing, vol. 18, no. 7, pp. 1452$1466,2009$. 
[15] C. Brito-Loeza and K. Chen, "On high-order denoising models and fast algorithms for vector-valued images," IEEE Transactions on Image Processing, vol. 19, no. 6, pp. 1518-1527, 2010.

[16] T. Howlader and Y. P. Chaubey, "Noise reduction of cDNA microarray images using complex wavelets," IEEE Transactions on Image Processing, vol. 19, no. 8, pp. 1953-1967, 2010.

[17] T. Mélange, M. Nachtegael, and E. E. Kerre, "Fuzzy random impulse noise removal from color image sequences," IEEE Transactions on Image Processing, vol. 20, no. 4, pp. 959-970, 2011.

[18] D. Zhai, M. Hao, and J. M. Mendel, "A non-singleton interval type-2 fuzzy logic system for universal image noise removal using quantum-behaved particle swarm optimization," in Proceedings of the IEEE International Conference on Fuzzy Systems (FUZZ '11), pp. 957-964, Taipei, Taiwan, June 2011.

[19] M. E. Yuksel and A. Basturk, "Application of type-2 fuzzy logic filtering to reduce noise in color images," IEEE Computational Intelligence MagazIne, vol. 7, no. 3, pp. 25-35, 2012.

[20] F. Russo, "New method for performance evaluation of grayscale image denoising filters," IEEE Signal Processing Letters, vol. 17, no. 5, pp. 417-420, 2010.

[21] F. Russo, "Accurate tools for analyzing the behavior of impulse noise reduction filters in color images," Journal of Signal and Information Processing, Scientific Research Publishing, vol. 4, pp. 42-50, 2013.

[22] N. Ponomarenko, F. Battisti, K. Egiazarian, J. Astola, and V. Lukin, "Metrics performance comparison for color image database," in Proceedings of the 4th International Workshop on Video Processing and Quality Metrics for Consumer Electronics, Scottsdale, Ariz, USA, January 2009.

[23] D. M. Chandler, "Seven challenges in image quality assessment: past, present, and future research," ISRN Signal Processing, vol. 2013, Article ID 905685, 53 pages, 2013.

[24] F. Russo, A. De Angelis, and P. Carbone, "A vector approach to quality assessment of color images," in Proceedings of the IEEE International Instrumentation and Measurement Technology Conference (I2MTC '08), pp. 814-818, Victoria, Canada, May 2008.

[25] A. De Angelis, A. Moschitta, F. Russo, and P. Carbone, "A vector approach for image quality assessment and some metrological considerations," IEEE Transactions on Instrumentation and Measurement, vol. 58, no. 1, pp. 14-25, 2009.

[26] “Kodak Lossless True Color Image Suite:” http://r0k.us/graphics/kodak/.

[27] A. Medda and V. DeBrunner, "Color image quality index based on the UIQI," in Proceedings of the 7th IEEE Southwest Symposium on Image Analysis and Interpretation, pp. 213-217, March 2006. 

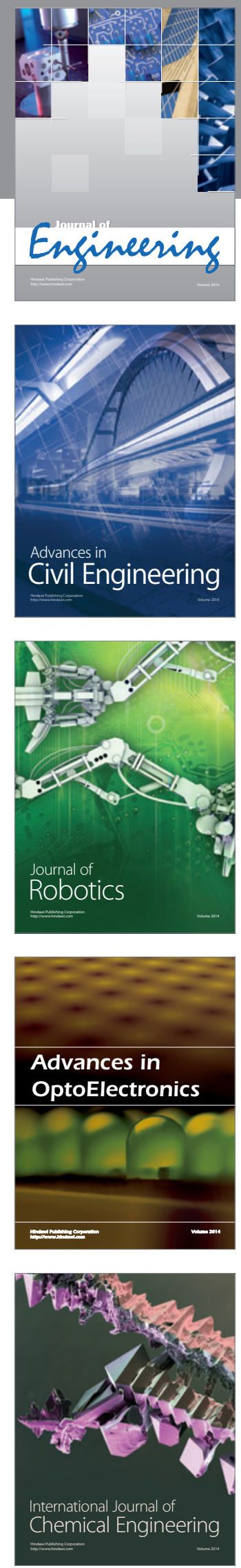

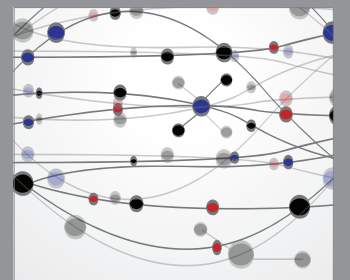

The Scientific World Journal
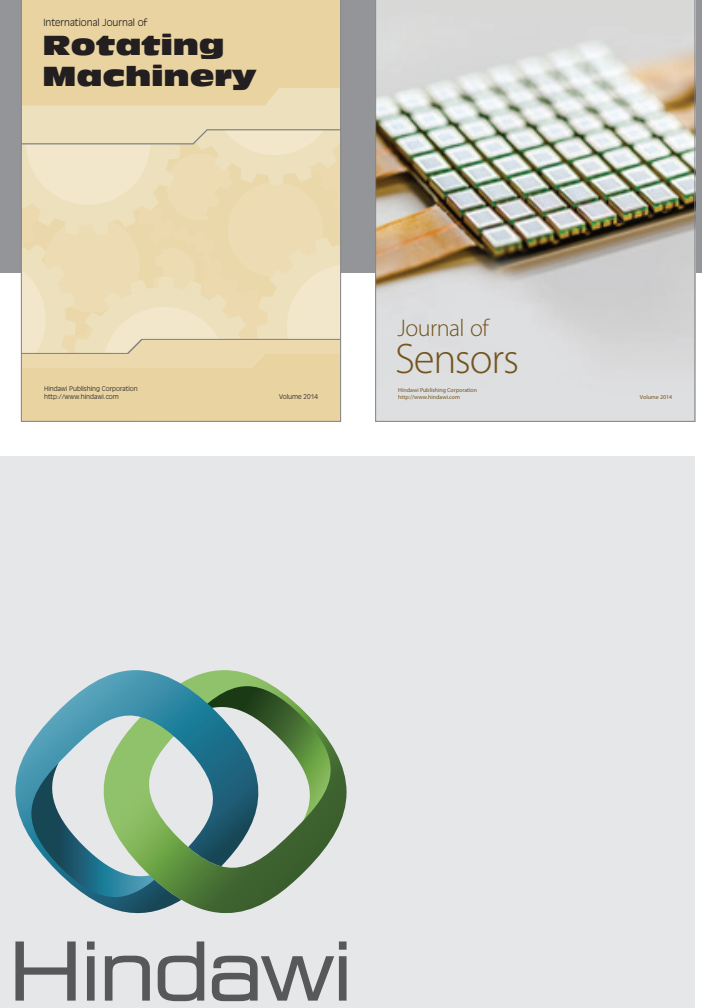

Submit your manuscripts at http://www.hindawi.com
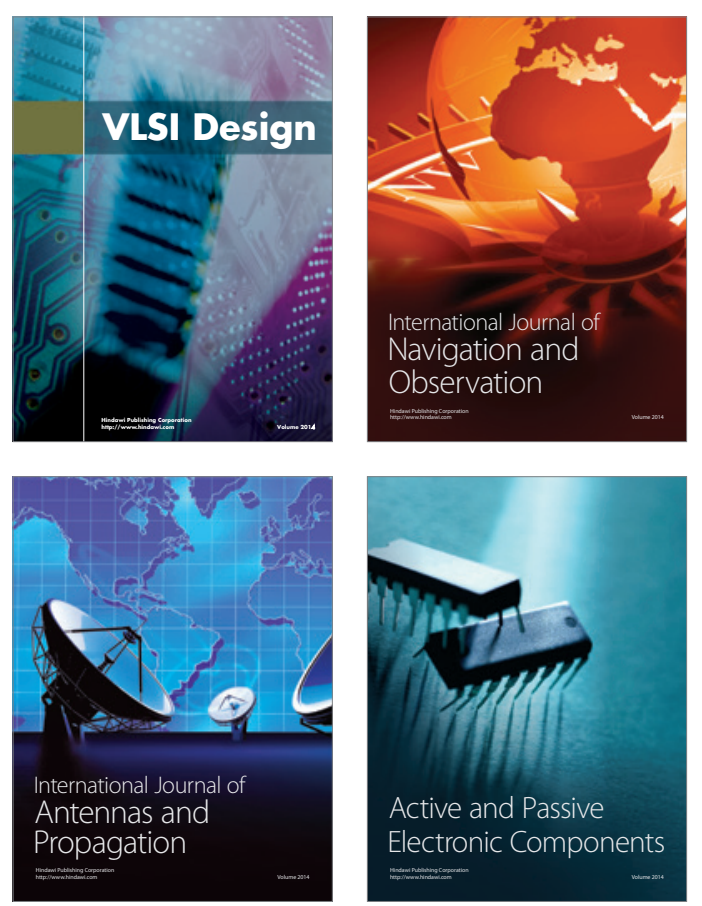
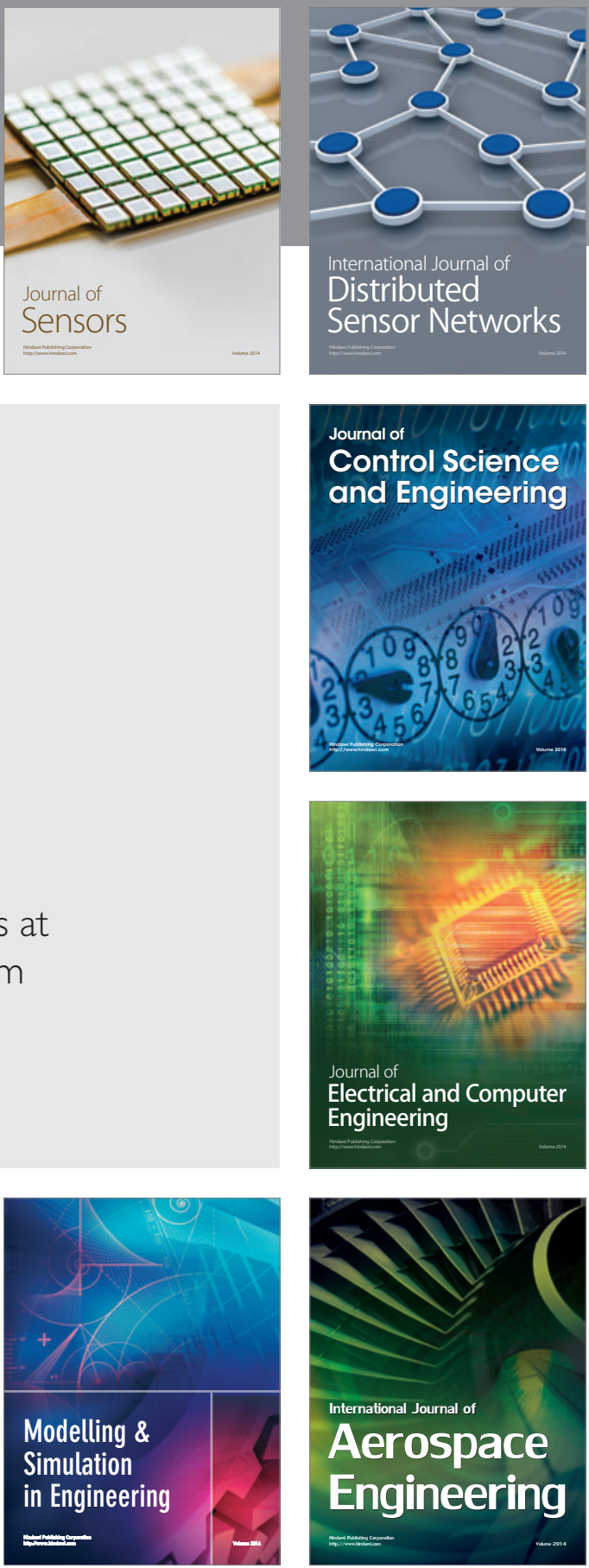

Journal of

Control Science

and Engineering
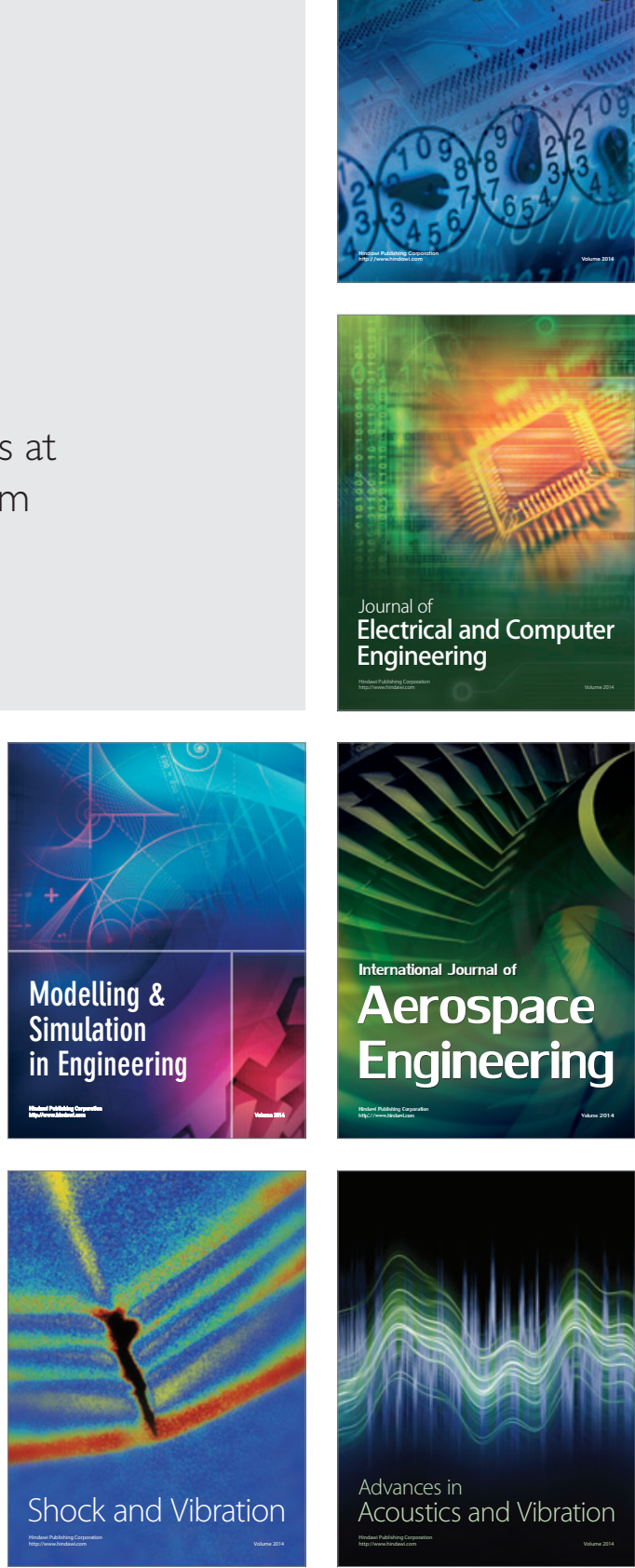